Commun. Math. Phys. 144, 337-350 (1992)

Communications in

Mathematical

Physics

(C) Springer-Verlag 1992

\title{
Classification of Singular Sobolev Connections by Their Holonomy
}

\author{
L. M. Sibner ${ }^{1, \star}$ and R. J. Sibner ${ }^{2, \star \star}$ \\ 1 Department of Mathematics, Polytechnic University, Brooklyn, NY 11201, USA \\ 2 Department of Mathematics, Brooklyn College, City University of New York, Brooklyn, \\ NY 11210, USA
}

Received June 9, 1989; in revised form August 5, 1991

\begin{abstract}
For a connection on a principal $S U(2)$ bundle over a base space with a codimension two singular set, a limit holonomy condition is stated. In dimension four, finite action implies that the condition is satisfied and an a priori estimate holds which classifies the singularity in terms of holonomy. If there is no holonomy, then a codimension two removable singularity theorem is obtained.
\end{abstract}

\section{Introduction}

Since the appearance of the fundamental paper of Uhlenbeck $\left[\mathrm{U}_{1}\right]$, apparent point singularities in gauge field theories have been studied extensively and are now fairly well understood $\left[\mathrm{O}_{1}, \mathrm{OS}, \mathrm{P}, \mathrm{S}, \mathrm{SS}_{1}, \mathrm{Sm}, \mathrm{U}_{1}, \mathrm{U}_{2}\right]$. The next step in this development concerns singular sets of higher dimension. If the singular set has codimension three or higher, the techniques used for point singularities (subelliptic estimates and broken Hodge gauges) can still be applied $\left[\mathrm{O}_{2}\right]$. Alternatively, these higher co-dimensional results, as well as the original removable point singularity theorems, can be obtained by straightforward modifications of the results in Sect. 5. On the other hand, co-dimension two singular sets, such as an $S^{2}$ embedded in $S^{4}$, introduce new geometric difficulties. These arise mainly from the fact that the complement of the singular set is no longer simply connected. Geometrically, this means that there are non-trivial flat connections. These connections cannot, in general, be extended to a neighborhood of the singular set even though the bundle itself may be topologically trivial. The appearance of holonomy is the obstacle to this extension. Physically, these singular sets give rise to finite action connections satisfying field equations whose topological charge is not integral but depends critically on holonomy $\left[\mathrm{A}, \mathrm{B}, \mathrm{C}, \mathrm{FHP}_{1}, \mathrm{FHP}_{2}\right]$.

$\star$ Research partially supported by NSF Grant DMS-8701813

$\star \star$ Research partially supported by NSF Grant INT-8511481 
In the following, for definiteness, we restrict our attention to four dimensions and singular sets $\Sigma$ which are smoothly embedded, orientable, connected 2-manifolds. Denote by $N$ a normal neighborhood of $\Sigma$ and let $X=N \backslash \Sigma$. We consider connections $D=d+A$ on $S U(2)$ bundles over $X$ which are "Sobolev" in the sense that $A \in L_{1, \text { loc }}^{2}(X)$ and the curvature $F=d A+A \wedge A$ belongs to $L^{2}(N)$. A holonomy condition is formulated for these connections $[(\mathrm{H})$ of Sect. 3].

Our first main result, Theorem 4.1, states that finite action implies the holonomy condition is satisfied, which answers a question raised by Atiyah in the course of these investigations. Our second result, Theorem 5.1, is an a priori estimate for finite action Sobolev connection which classifies their singularities completely in terms of holonomy. The ideas involved in proving this estimate are those of Taubes. Under certain conditions on the holonomy, this results in a codimension two removable singularity theorem which we state as Theorem 5.2. An important interesting special case is concerned with connections which admit an $S^{1}$ action about the singular set. In Sect. 2 we review Atiyah's construction of hyperbolic monopoles via dimensional reduction. (This correspondence between monopoles on a hyperbolic 3-manifold and $S^{1}$ invariant instantons on a 4-manifold has been exploited by Braam [B].) In this context, Corollary 5.3 shows that the Prasad-Sommerfield limit for the finite action Higgs model over $H^{3}$ exists at infinity.

In joint work with $\mathrm{K}$. Uhlenbeck, the above results are used to show the existence of non-self-dual Yang-Mills fields in $S U(2)$ bundles over $S^{4}$ [SSU].

\section{Hyperbolic Monopoles}

Following Atiyah [A], we briefly recall the relationship between $S^{1}$ invariant instantons on $R^{4}$ and magnetic monopoles on $H^{3}$.

Locally, choose coordinates $(u, v, r, \theta)$ with $(u, v)$ coordinates on $\Sigma=R^{2}$ and $(r, \theta)$ coordinates in planes normal to $\Sigma$. The Euclidean metric element

$$
d s^{2}=r^{2}\left\{\frac{d u^{2}+d v^{2}+d r^{2}}{r^{2}}+d \theta^{2}\right\}
$$

exhibits a conformal equivalence:

$$
R^{4} \backslash R^{2} \sim H^{3} \times S^{1},
$$

where $H^{3}$ is hyperbolic 3-space with constant curvature -1 .

On $R^{4}$, the self-dual Yang-Mills (instanton) equations are

$$
* F=F \text {, }
$$

where $F$ is the curvature form of a connection.

On $H^{3}$, the Bogomol'nyi (monopole) equations are

$$
D \phi=* F^{\prime} \text {, }
$$

where $F^{\prime}$ is a curvature form and $\phi$ (the Higgs field) is a section of the adjoint bundle.

Given a rotationally invariant instanton (independent of $\theta$ ) on $R^{4} \backslash R^{2}=S^{4} \backslash S^{2}$ it descends via dimensional reduction (see $[\mathrm{A}, \mathrm{JT}]$ ) to a hyperbolic monopole on $\mathrm{H}^{3}$. The Higgs field $\phi=A_{\theta}$, and, letting $\theta=x^{4}, F^{\prime}=\frac{1}{2} \sum_{i, j=1}^{3} F_{i j} d x^{i} \wedge d x^{j}$. Finite action is 
preserved and the second Chern class $c_{2}$ of the bundle over $S^{4} \backslash S^{2}$ satisfies

$$
c_{2}=\frac{1}{8 \pi^{2}} \int|F|^{2} d x^{4}=\frac{1}{4 \pi} \int\left(\left|F^{\prime}\right|^{2}+|D \phi|^{2}\right) d x^{3}=\frac{2}{4 \pi} \int\left|F^{\prime}\right|^{2} d x^{3}=2 k m .
$$

The integer $k$, the magnetic charge of the monopole, is the winding number of $\phi$, or the number of zeros of the Higgs field $\phi$. The number $m$, the mass of the monopole, is the asymptotic value of $|\phi|$ at infinity. As we shall later see, $m$ completely describes the holonomy of the connection about the singular set in $R^{4}$. A necessary condition that a connection extend to all of $R^{4}$ is that $m$ is an integer.

\section{Properties of Holonomy}

For $\varepsilon$ sufficiently small, a normal $\varepsilon$-neighborhood $N$ of $\Sigma$ is locally a product and it is convenient to choose local coordinates in the following way. Restricting to a coordinate chart $\Sigma_{0} \subset \Sigma$ (and corresponding $N_{0}$ ) we obtain coordinates $(u, v)$ on $\Sigma_{0}$ and polar coordinates $(r, \theta)$ in the normal plane to $\Sigma_{0}$ at $(u, v)$. Setting $X_{0}=N_{0} \backslash \Sigma_{0}$, a standard topological argument shows that any $S U(2)$ bundle over $X_{0}$ is trivial.

We next describe the phenomenon of holonomy, first discussed by Taubes in this context. Given a connection on an $S U(2)$ bundle over $X_{0}$, parallel transport of the fibers around a generator of $\pi_{1}\left(X_{0}\right)$ gives an automorphism of the fiber which we realize in the usual way as an element of $S U(2)$ as follows. Write the connection $D=d+A$ where, using the above coordinates, $A=A_{u} d u+A_{v} d v+A_{r} d r+A_{\theta} d \theta$. In a fixed normal plane, the initial value problem.

$$
\frac{d g_{r}}{d \theta}+A_{\theta} g_{r}=0, \quad g_{r}(0)=I
$$

has a unique solution $g_{r}(\theta)$. We will not need an explicit representation of the solution which would be given by a path ordered integral (cf. [IZ]). [If $D$ is $S^{1}$ invariant with $A_{\theta}$ independent of $\theta$, then $g_{r}(\theta)$ is obtained, as in the scalar case, by exponentiating.]

Set $g_{r}(2 \pi)=J_{r} \in S U(2)$. The conjugacy class $\left[J_{r}\right]$ of the "phase factor" $J_{r}$ is gauge invariant. For completeness, we include a proof based on Eq. (3.1).

Lemma 3.2. Let $\mathrm{g}_{\mathrm{r}}$ and $\hat{\mathrm{g}}_{\mathrm{r}}$ be solutions of (3.1) for gauge equivalent connections $D=d+A$ and $\hat{D}=d+\hat{A}$. Then $g_{r}(2 \pi)$ and $\hat{\mathrm{g}}_{r}(2 \pi)$ are conjugate in $S U(2)$.

Proof. Let $s: X_{0} \rightarrow S U(2)$ be the gauge transformation such that $\hat{A}=s^{-1} A s+s^{-1} d s$. Continuity of $s$ requires that $s$ be periodic in $\theta, s(0)=s(2 \pi)$. Solving (3.1) in the new gauge

$$
\frac{d \hat{\mathrm{g}}_{r}}{d \theta}+\left(s^{-1} A_{\theta} s+s^{-1} \frac{d s}{d \theta}\right) \hat{\mathrm{g}}_{\mathrm{r}}=0, \quad \hat{\mathrm{g}}_{r}(0)=I
$$

it is easy to see that the function $s(\theta) \hat{g}_{r}(\theta) s^{-1}(0)$ solves (3.1) in the original gauge. By uniqueness, it must then equal $g_{r}(\theta)$ and hence $g_{r}(2 \pi)=s(2 \pi) \hat{g}_{r}(2 \pi) s^{-1}(2 \pi)$, proving the lemma.

If the connection were flat, then the holonomy $\left[J_{r}\right]$ would be a homotopy invariant and hence independent of $r$. In general, we can ask that a "limit" holonomy exists as we shrink to a point $P$ on the 2 -manifold $\Sigma$. We state this as the 
(H) Limit Holonomy Condition:

$\lim _{r \rightarrow 0}\left[J_{r}\right]=[J]$ exists for almost all $P \in \Sigma$, and is independent of $P$.

We will show in Sect. 4, under the rather mild assumption of finite action, that the holonomy condition is satisfied and see, in Sect. 5 , how this implies that the given connection is, near the singular 2-manifold, "asymptotic" to a flat connection with the same holonomy. It follows immediately from Lemma 3.2 that the condition $(\mathrm{H})$ is gauge invariant for smooth gauge transformations. In fact

Remark. Lemma 3.2 holds if $A$ and $\hat{A}$ are related by a "weak" gauge transformation $s \in L_{2, l_{\mathrm{c}} \mathrm{c}}^{2}$ Thus $A$ satisfies condition $(\mathrm{H})$ if it is weakly gauge equivalent to a connection $A$ for which $\left[\hat{J}_{r}\right]$ has a limit.

Next we compile some information about flat bundles which will be needed in the analysis which follows. Recall that for any $Y$ with $\pi_{1}(Y)=Z$, the flat (zero curvature) $S U(2)$ bundles over $Y$ are in 1-1 correspondence with conjugacy classes in $S U(2)$. Such conjugacy classes can be uniquely described by their traces which take values in some finite interval of the real line. Choosing the diagonal representative

$$
\left(\begin{array}{cc}
e^{-2 \pi i m} & 0 \\
0 & e^{2 \pi i m}
\end{array}\right)
$$

in each conjugacy class, we can identify every flat bundle with a real number $m$.

On the other hand, the prototype of a flat connection over $Y$ is given by

$$
A^{b}=\left(\begin{array}{cc}
i m & 0 \\
0 & -i m
\end{array}\right) d \theta=m \hat{i d} \theta
$$

with $m \hat{i} \in S u(2)$.

The solution of (3.1) with $A_{\theta}=m \hat{i}$, is

$$
g(\theta)=\left(\begin{array}{cc}
e^{-i m \theta} & 0 \\
0 & e^{i m \theta}
\end{array}\right)
$$

and $g(2 \pi)$ is the diagonal element (3.3). For flat bundles, the solution of (3.1) is homotopy invariant and therefore independent of $r$. Hence, (3.3) and (3.4) exhibit a unique correspondence between holonomy and flat connections.

We conclude this section with two useful facts about these flat connections.

Lemma 3.6. If $m_{1}=m_{2}+n, n=$ integer, then $A_{1}^{b}$ and $A_{2}^{b}$ are gauge equivalent.

Proof. $A_{1}^{b}=A_{2}^{b}+g^{-1} d g$ with $g(\theta)=\left(\begin{array}{cc}e^{i n \theta} & 0 \\ 0 & e^{-i n \theta}\end{array}\right)$.

Corollary 3.7. If $m$ is an integer then $D$ is gauge equivalent to $d$.

It follows that, locally, flat bundles are indexed by $m$ belonging to some finite interval, for example, $0 \leqq m<1$.

Lemma 3.8. Let $D=d+A$ be a flat connection with holonomy $m$. Then, there is $a$ gauge in which $D=d+A^{b}$.

Lemma 3.8 follows from the previous discussion. These results apply with $Y=X_{0}=N_{0} \backslash \Sigma_{0}$ and also with $Y=a$ punctured normal disk in $X_{0}$. 


\section{A Sufficient Analytic Condition for Holonomy}

In this section, we give sufficient analytic conditions on Sobolev connections which insure that condition $(\mathrm{H})$ is satisfied. We formulate these as

Theorem 4.1 (Existence of Limit Holonomy). Let $N$ be a normal $\varepsilon$-neighborhood of the 2-dimensional submanifold $\Sigma$ of a 4-manifold $M$. If $D=d+A$ with $A \in L_{1,10 c}^{2}(N \backslash \Sigma)$ and $F \in L^{2}(N)$ then the holonomy limit $(\mathrm{H})$ of $D$ at $\Sigma$ exists. Locally, there is a gauge in which the components of $A$ have a limit at $\Sigma$ with, in particular, $A_{\theta} \rightarrow$ mi a.e.

Proof. In $X_{0}=N_{0} \backslash \Sigma_{0}$ we "gauge away" $A_{r}$, the radial component of $A$, by solving (in the weak sense)

$$
\frac{d g}{d r}+A_{r} g=0 \quad \text { with } \quad g\left(u, v, \varrho_{0}, \theta\right)=I, \quad \varrho_{0}>0 .
$$

Since $A \in L_{1, \text { loc }}^{2}$, we have $g \in L_{2, \text { loc }}^{2}$. By Fubini's theorem, $g$ is continuous on almost all normal planes and, moreover, is periodic in $\theta$ of period $2 \pi$. In the new "transverse" gauge with $A_{r}=0$,

$$
\frac{\partial A_{\theta}}{\partial r}=F_{r \theta} .
$$

In a Fourier series expansion,

$$
A_{\theta}=\sum_{n=-\infty}^{\infty} a_{n}(r) e^{i n \theta} .
$$

Again, by Fubini's theorem, $F$ is in $L^{2}$ on almost all normal planes. Choosing a normal plane at $\left(u_{0}, v_{0}\right)$ and using $(4.3)$, we obtain

$$
\int_{r_{1}}^{r_{2}} \sum_{n=-\infty}^{\infty}\left|\frac{\partial a_{n}}{\partial r}\right|^{2} d r \leqq k \int_{\substack{j_{1} \leqq r \leq r_{2} \\ u=u_{0} \\ v=v_{0}}}|F|^{2} r d r d \theta .
$$

Letting $r_{k}$ be a sequence of radii tending to zero, the corresponding sequence of Fourier series (4.4) forms a Cauchy sequence and hence converges to a limit.

$$
c_{\theta}=\sum_{n=-\infty}^{\infty} a_{n}(0) e^{i n \theta} .
$$

The 1-form $c=c_{\theta} d \theta$ defines a flat connection on the normal plane through $\left(u_{0}, v_{0}\right)$. From Lemma $3.8 c$ is gauge equivalent to an $A^{b}$ of the form (3.4). Therefore,

$$
s^{-1} c s+s^{-1} d s=A^{b}=m \hat{i d} \theta,
$$

where $s$ depends only on $\theta$. Varying $u$ and $v$, we can extend $s$ to all of $X_{0}$ so that the extension is still independent of $r$.

We now return to the gauge in which $A_{r}=0$ and gauge transform by $s$ to obtain $\hat{A}=s^{-1} A s+s^{-1} d s$ with $\hat{A}_{r}=0$. An inequality of the form (4.5) then holds for the Fourier coefficients of $\hat{A}_{\theta}$, and therefore, $\hat{A}_{\theta}$ converges to a limit as $r \rightarrow 0$. However, $\hat{A}_{\theta}=s^{-1} A_{\theta} s+s^{-1} \frac{d s}{d \theta}$ converges to $s^{-1} c_{\theta} s+s^{-1} \frac{d s}{d \theta}$ and $\hat{A}_{\theta} d \theta$ converges to $A^{\mathrm{b}}$. It 
follows from continuous dependence on parameters, that the solution of the initial value problem (3.1) converges. Therefore, the limit in $(\mathrm{H})$ exists.

Next, we show that the limit in $(\mathrm{H})$ is independent of the normal plane. Let $P_{1}$ and $P_{2}$ be two points of $\Sigma$ at which the limit exists. By arranging coordinates, we can always assume that $P_{1}$ and $P_{2}$ are contained in a single coordinate chart $\Sigma_{0}$ and that $P_{1}=\left(u_{1}, v_{0}\right)$ and $P_{2}=\left(u_{2}, v_{0}\right)$.

There exists a sequence of cylinders $C_{i}=\left\{\left(u, v_{0}, r_{i}, \theta\right) \mid u_{1} \leqq u \leqq u_{2}, 0 \leqq \theta \leqq 2 \pi\right\}$ with $r_{i}$ tending to zero such that, by Fubini's theorem,

$$
r_{i} \iint_{C_{i}}|F|^{2} d S \rightarrow 0
$$

Choose a gauge in which $A_{u}=0$ so that $\frac{\partial A_{\theta}}{\partial u}=F_{u \theta}$. Then

$$
\int_{0}^{2 \pi}\left|A_{\theta}\left(u_{2}, v_{0}, r_{i}, \theta\right)-A_{\theta}\left(u_{1}, v_{0}, r_{i}, \theta\right)\right| d \theta \leqq k r_{i} \int_{C_{r_{i}}}|F|^{2} d S .
$$

Denote by $g_{1}$ and $g_{2}$ the solutions of (3.1), at $r=r_{i}$, in the normal planes at $\left(u_{1}, v_{0}\right)$ and $\left(u_{2}, v_{0}\right)$, respectively. Using the fact that $g_{1}$ is a solution of

$$
\frac{d g_{1}}{d \theta}+A_{\theta}\left(u_{2}, \cdot\right) g_{1}=\left(A_{\theta}\left(u_{2}, \cdot\right)-A_{\theta}\left(u_{1}, \cdot\right)\right) g_{1}, \quad g_{1}(0)=I,
$$

elementary computations show that

$$
\left|g_{2}^{-1}(2 \pi) g_{1}(2 \pi)-I\right| \leqq k r_{i} \int_{C_{i}}|F|^{2} d S \rightarrow 0
$$

which proves that the holonomy limit is the same at $P_{1}$ and $P_{2}$. This completes the proof of Theorem 4.1.

\section{An a priori Estimate for Finite Action Connections}

In this section we prove our main estimate for Sobolev connections. Again, $N_{0}$ is a normal $\varepsilon$-neighborhood of the coordinate chart $\Sigma_{0}$ and $X_{0}=N_{0} \backslash \Sigma_{0}$. The underlying topology is that of the space

$$
\mathscr{A}^{p}=\left\{D=d+A \mid A \in L_{1, \text { loc }}^{p}\left(X_{0}\right) \text { and } F \in L^{p}\left(N_{0}\right)\right\}
$$

with $p \geqq 2$. It follows from Theorem 4.1 that if $D \in \mathscr{A}^{p}$, a holonomy condition $\left(H_{m}\right)$ is satisfied and this determines a constant flat connection $A^{b}$ of the form mid $\theta$.

Given any flat connection $\Gamma$ with holonomy $m$, we consider the space $L_{1, \Gamma}^{p}$ of 1 -forms $\omega$ with finite $\Gamma$ norm

$$
\|\omega\|_{L_{1, \Gamma}^{p}}=\|\omega\|_{L^{p}}+\left\|\nabla_{\Gamma} \omega\right\|_{L^{p}}<\infty,
$$

where $\nabla_{\Gamma}$ is the covariant derivative at $\Gamma$.

We can now state our main result.

Theorem 5.1 (Classification by Holonomy). There exists a constant $k>0$ such that, for $D \in \mathscr{A}^{2}$ with $\|F\|_{L^{2}\left(N_{0}\right)}<k$ there is a real number $m$ (with corresponding flat connection $A^{b}=$ mid $\theta$ ) and a gauge in which $D=d+A$ with $A-A^{b} \in L_{1, A^{b}}^{2}\left(N_{0}\right)$. Moreover, for some constant $C$,

$$
\left\|A-A^{b}\right\|_{L_{1, A^{b}}^{2}} \leqq C\|F\|_{L^{2}} .
$$


Remark. One might hope to obtain a co-closed connection in the gauge orbit of $D$ and hence, a "slice theorem" by using the implicit function theorem. However, an example of Taubes shows that such a result cannot be true in general if $2 m$ is not an integer.

Since for $m \in Z$ the flat connection $A^{b}=m \hat{i} d \theta$ is gauge equivalent to the zero connection, we obtain the following important corollary.

Theorem 5.2 (Codimension Two Removable Singularity). If the connection D satisfies the hypothesis of Theorem 5.1 with holonomy $m$ an integer, then $D$ extends as an $L_{1}^{2}$ connection to all of $N_{0}$. If field equations are satisfied, more smoothness follows from elliptic theory.

If $D$ represents an $S^{1}$ invariant, finite action instanton over $R^{4} \backslash R^{2}$ as in Sect. 2, we obtain an estimate for the Higgs field $\phi$ of the associated hyperbolic monopole; namely, from inequality (5.1), Sobolev's inequality, and conformal invariance of norms, we have

Corollary 5.3. If $\left(A^{\prime}, \phi\right)$ is a finite action Yang-Mills-Higgs configuration with field strength $F^{\prime}$, on hyperbolic 3-space $H^{3}$, then there exist a constant $k$ such that

$$
\||\phi|-m\|_{L^{4}\left(H^{3}\right)} \leqq k\left\|A-A^{b}\right\|_{L_{1}^{2}\left(S^{4}\right)} \leqq 2 k C\left\|F^{\prime}\right\|_{L^{2}\left(H^{3}\right)} .
$$

The construction in Sect. 2 produces a hyperbolic monopole on $H^{3}$ given an $S^{1}$-invariant instanton on $S^{4}$. Conversely, we obtain

Corollary 5.4. A finite action monopole on $H^{3}$ whose mass $m$ is an integer corresponds to an $S^{1}$-invariant, finite action instanton over all of $S^{4}$.

The rest of this section is devoted to the proof of Theorem 5.1 in which we carry out the details of Taubes' program. We cover the space $X_{0}=N_{0} \backslash \Sigma_{0}$ by a countable collection of balls. On each ball, we have a "good" gauge, namely the Hodge (or Coulomb) gauge obtained from Uhlenbeck's theorem $\left[\mathrm{U}_{2}\right]$, in which the Sobolev norm of the connection form can be estimated by curvature. These gauges are then glued together to produce a global gauge. The contributions to the connection form which cannot be controlled by curvature are then seen to arise from holonomy and these terms define a flat connection.

Let

$$
\mathscr{A}_{m, k}^{p}=\left\{D \in \mathscr{A}^{p} \mid\|F\|_{L^{2}} \leqq k \text { and }\left(H_{m}\right) \text { holds }\right\} .
$$

Our main technical result is

Proposition 5.5. Let $p>2$. Then, there exist $k$ and $\hat{c}$, and an explicit flat connection $\Gamma$ with holonomy m, such that if $D \in \mathscr{A}_{m, k}^{p}$, then there is a gauge in which $D=d+\widehat{A}$ and

$$
\|\hat{A}-\Gamma\|_{L_{1, \Gamma}^{q}\left(N_{0}\right)} \leqq \hat{c}\|F\|_{L^{q}\left(N_{0}\right)}, \quad 2 \leqq q \leqq p .
$$

From this we obtain

Corollary 5.6. There exists a gauge in which $D=d+A$ with $A-A^{b} \in L_{1, A^{b}}^{p}\left(N_{0}\right)$ and for $2 \leqq q \leqq p$,

$$
\left\|A-A^{b}\right\|_{L_{1, A^{b}\left(N_{0}\right)}^{q}} \leqq C\|F\|_{L_{\left(N_{0}\right)}} .
$$

Corollary 5.6 implies Theorem 5.1. Approximate $\tilde{A}$ in $L_{1, \text { loc }}^{2}$ by smooth $\tilde{A}_{j}$ with $\left\|F_{j}\right\|_{L^{2}\left(N_{0}\right)}<k$. Apply Corollary 5.6 to each $\tilde{A}_{j} \in L_{1, \text { loc }}^{p}, p>2$. For each $D_{j}$, there is a 
gauge in which $D_{j}=d+A_{j}$, where $A_{j}$ satisfies (5.6) with some $A_{j}=m_{j} \hat{i} d \theta$. The bound in (5.6) with $q=2$ implies that a subsequence of $A_{j}-A_{j}^{b}$ converges weakly in $L_{1}^{2}$ to some $A-A_{\infty}^{b}$. For each $j, s_{j}^{-1} \tilde{A}_{j} s_{j}+s_{j}^{-1} d s_{j}=A_{j}$. It follows that a diagonal subsequence of $\left\{s_{j}\right\}$ converges weakly to $s \in L_{2, \text { loc }}^{2}$. In the limit,

$$
d s=s A-\tilde{A} s
$$

with $s \in L_{2, \text { loc }}^{2}\left(X_{0}\right)$. From the remark following Lemma 3.2, $A$ and $\tilde{A}$ have the same holonomy. It follows that $\widetilde{A}$ is weakly gauge equivalent to $A$ satisfying (5.6) which proves Theorem 5.1.

We now turn to the proof of Proposition 5.5. The proof involves the basic theorem of Uhlenbeck concerning the existence of Coulomb gauges and several technical lemmas.

We cover $X_{0}=N_{0} \backslash \Sigma_{0}$ by balls $B_{\alpha}$ in each of which a good gauge is given by Theorem 5.7 (Uhlenbeck $\left[\mathrm{U}_{2}\right]$ ). For $k$ sufficiently small, there is a gauge in which $D=d+A^{\alpha}$ in $B_{\alpha}$ with

$$
\begin{gathered}
d^{*} A^{\alpha}=0, \\
\left\|A^{\alpha}\right\|_{L_{1}^{q}\left(B_{\alpha}\right)} \leqq K\|F\|_{L^{q}\left(B_{\alpha}\right)}, \quad 2 \leqq q \leqq p, \\
\frac{1}{\operatorname{Vol} B_{\alpha}} \int_{B_{\alpha}} A^{\alpha} d V=0 .
\end{gathered}
$$

Conditions a and $\mathrm{b}$ are in $\left[\mathrm{U}_{2}\right]$. To prove c, note that in the Abelian case, $A^{\alpha}$ is gauge equivalent to $A=A^{\alpha}+d u_{0}$ with $u_{0}=C x$ and $C$ chosen so that $A$ has mean value zero. In the non-Abelian case, $A$ may be found by iterating with $s_{0}=e^{u_{0}}=e^{C x}$, $s_{n}=e^{u_{n}}$ and solving $\Delta u_{n}=e^{-u_{n-1}}\left[A^{\alpha}, d u_{n-1}\right] e^{u_{n-1}}$ with $\int_{B_{\alpha}} d u_{n}=-\int_{B_{\alpha}} e^{-u_{n-1}} A^{\alpha} e^{u_{n-1}}$. Since, $\left\|u_{n}\right\|_{L_{2}^{2}\left(B_{\alpha}\right)} \leqq\left\|A^{\alpha}\right\|_{L_{1}^{2}\left(B_{\alpha}\right)} \leqq k\|F\|_{L^{2}\left(B_{\alpha}\right)}$, we see that $u_{n}$ converges to $u$ with $\|u\|_{L_{2}^{2}\left(B_{\alpha}\right)} \leqq k\|F\|_{L^{2}\left(B_{\alpha}\right)}$. Therefore, $A=e^{-u} A^{\alpha} e^{u}+d u$ satisfies the conditions a, b, c.

Remark. The connection $A^{\alpha}$ is not uniquely specified but can be altered by a constant gauge transformation. This gauge freedom will be used in gluing gauges together.

First, we describe the precise covering constructed by Taubes and then, his definition of the global gauge transformation.

We assume that $\left\{B_{\alpha}\right\}$ is an open covering of $X_{0}$ which contains a subcovering $\left\{U_{\alpha}\right\}$ defined in the following manner. Let $\Sigma_{0}$, the local singular set, be diffeomorphic to a square in $R^{2}$. The index $\alpha=(n, l, x)$ where $n$ is a positive integer, $l$ takes integer values from 0 to 7 , and $x$ is a point of $\Sigma_{0}$ lying on the standard square lattice $\Lambda_{n}$ of side length $2^{-n-1}$. With this notation, $U_{\alpha}$ consists of the points with coordinates $(r, \theta, y)$ restricted as follows:

(i) $2^{-n-2}<r<2^{-n}$,

(ii) $\frac{\pi l}{4}<\theta<\frac{\pi}{4}(l+2)$,

(iii) $y$ belongs to the open square centered at $x$ of the length $2^{-n}$.

On intersections $U_{\alpha} \cap U_{\beta}$, the Coulomb connections are related by the gauge transformations $A^{\beta}=e^{-u} A^{\alpha} e^{u}+d u$ where we have written $e^{u}=h_{\alpha}^{-1} h_{\beta}$. Therefore, we obtain 
Corollary 5.8. $O n U_{\alpha} \cap U_{\beta}$

$$
\frac{1}{d^{2}}\|u-u(P)\|_{L^{q}}+\frac{1}{d}\|\nabla u\|_{L^{q}}+\|\nabla(\nabla u)\|_{L^{q}} \leqq \operatorname{const}\left(\|F\|_{L^{q}\left(U_{\alpha}\right)}+\|F\|_{L^{q}\left(U_{\beta}\right)}\right),
$$

where $P$ lies in $U_{\alpha} \cap U_{\beta}$ and $\alpha$ is the larger of the diameters of the two balls $B_{\alpha}$ and $B_{\beta}$. Lemma 5.9. The function $u$ in Corollary 5.8 may be chosen to satisfy on $U_{\alpha} \cap U_{\beta}$ (with $\alpha=(n, l, x)$ and $\beta=\left(n^{\prime}, l^{\prime}, x^{\prime}\right)$ ) the constraints:

a) If $n=n^{\prime}, x=x^{\prime}$ and $l=l^{\prime}+1$ with $1 \leqq l^{\prime}<7$, then $u(P)=0$.

b) If $n=n^{\prime}, l=l^{\prime}$ and $x$ and $x^{\prime}$ are adjacent on the square lattice $\Lambda_{n}$, then $u(P)=0$.

c) If $n=n^{\prime}+1$ and $l=l^{\prime}$ and $x=x^{\prime}$, then $u(P)=0$.

Proof. The gauge freedom can be used to achieve the above conditions. However, note the important fact that strict inequality holds in a) which means that $u(P)$ cannot be chosen to be zero in an intersection in which $l^{\prime}=7$.

We now proceed with Taubes definition of the global gauge transformation. Because of the non-Abelian nature of $S U(2)$, at each stage, at most two local gauge transformations can be glued together by a cutoff function. The strategy consists of four steps. First, the transition functions of Corollary 5.8 are glued together as $\theta$ goes from 0 to 7, with $n$ and $x$ fixed. Secondly, the resulting gauges corresponding to $x$ and $x^{\prime}$ with the same $n$ are glued together. Thirdly, the gauge indexed by $n$ is glued to the gauge indexed by $n+1$. This produces a gauge which is multivalued in the sense that $g(r, 2 \pi, y) \neq g(r, 0, y)$. The gauge transformation $s=g(r, 2 \pi, y)^{-1} g(r, 0, y)$ is first shown to have a pointwise limit as $r$ tends to zero which may be identified with the holonomy. Then, using a carefully normalized local radial gauge, $s$ can be shown to satisfy good a priori estimates. Finally, from this analysis, $s$ can be modified to obtain a global gauge in which the connection form satisfies the inequality of Proposition 5.5.

We begin by fixing a cutoff function

$$
\lambda(t)=\left\{\begin{array}{ccc}
1 & \text { for } & 0 \leqq t \leqq \frac{\pi}{16} \\
0 & \text { for } & \frac{3 \pi}{16} \leqq t \leqq 2 \pi
\end{array}\right.
$$

and let $\lambda_{l}(\theta)=\lambda\left(\theta-\frac{\pi l}{4}\right)$ be periodic in $\theta$ of period $2 \pi$. With $n$ and $x$ fixed, we define a gauge transformation on $\bigcup_{l=0}^{7} U_{(n, l, x)}$ by

$$
h_{n, x} \mid U_{(n, l, x)}=h_{(n, l, x)} \exp \left(\lambda_{l} u_{l}\right),
$$

where $\exp u_{l}=h_{(n, l, x)}^{-1} h_{(n, l-1, x)}$. Note that $h_{n, x}$ as defined on $U_{(n, 0, x)}$ does not agree with its definition on $U_{(n, 7, x)}$.

Secondly, move along the singular set from a point $x=\left(x_{1}, x_{2}\right)$ on the lattice $\Lambda_{n}$ to a neighboring point $x^{\prime}=\left(x_{1}, x_{2}-2^{-(n+1)}\right)$. Now, define

$$
\lambda(y)=\left\{\begin{array}{lll}
1 & \text { for } y \leqq x_{2}-2^{-(n+2)} \\
0 & \text { for } y \geqq x_{2}
\end{array}\right.
$$


and let $u\left(n, x, x^{\prime}\right)$ be defined by $\exp u\left(n, x, x^{\prime}\right)=h_{n, x}^{-1} h_{n, x^{\prime}}$. Then, on $U_{(n, l, x)} \cap U_{\left(n, l, x^{\prime}\right)}$, our global gauge transformation is given by

$$
h_{n}=h_{n, x} \exp \left(\lambda(y) u\left(n, x, x^{\prime}\right)\right) \text {. }
$$

To get from the lattice point $x=\left(x_{1}, x_{2}\right)$ to $x^{\prime \prime}=\left(x_{1}-2^{-(n+2)}, x_{2}\right), \lambda$ and $u$ are defined in an analogous way and on $U_{(n, l, x)} \cap U_{\left(n, l, x^{\prime \prime}\right)}$, the definition of $h_{n}$ is clear.

The third step is now to move from $n$ to $n+1$. To this end, let

$$
\lambda(r)= \begin{cases}0 & \text { for } r \leqq 2^{-(n+1)} \\ 1 & \text { for } r \geqq \frac{3}{2} 2^{-(n+1)}\end{cases}
$$

Then, on the appropriate intersection on which $\exp u=h_{n}^{-1} h_{n+1}$, we define

$$
g=h_{n} \exp (\lambda u) \text {. }
$$

It follows from Corollary 5.8, that each of the $u$ 's occurring on an intersection belongs to $L_{2}^{q}$ for $q>2$, and hence, to $L^{\infty}$ by Sobolev's lemma. Moreover, using the gauge freedom of Lemma 5.9, which allows the choice of a gauge for which $u(P)=0$, we find that in all intersections except those involving $l=0$ and $l=7$,

$$
\frac{1}{d^{2}}\|u\|_{L^{q}}+\frac{1}{d}\|\nabla u\|_{L^{q}}+\|\nabla(\nabla u)\|_{L^{q}} \leqq \operatorname{const}\left(\|F\|_{L^{q}\left(U_{\alpha} \cup U_{\beta}\right)}\right) .
$$

Using the basic facts about cutoff functions that $|\nabla \lambda| \leqq c d$ and $|\nabla(\nabla \lambda)| \leqq c d^{2}$, we find that for $g$ defined above, on an intersection not involving $\theta=0$ and $\theta=2 \pi$, the one form $\omega=g^{-1} d g \in L_{1}^{q}$ obeys

$$
\|\omega\|_{L_{1}^{q} \leqq \text { const }\|F\|_{L^{q}\left(U_{\alpha} \cup U_{\beta}\right)}} .
$$

The fourth and final step in the proof is to deal with the multi-valuedness of $g$.

Let $g_{0}=g \mid U_{(n, 0, x)}$ and $g_{7}=g \mid U_{(n, 7, x)}$. Then, the gauge transformation $s=g_{0}^{-1} g_{7}$ is defined on $\bigcup_{(n, x)}\left\{U_{(n, 0, x)} \cap U_{(n, 7, x)}\right\}$.

We next prove a theorem of Taubes which shows directly that $s$ has a limit as $n$ tends to infinity which is independent of $x$. Finally, to prove the inequality of Proposition 5.5, with $p>2$, we use some very precise information obtained from a well normalized radial gauge.

Define the set $V=V[n, x]=\{(r, \theta, y)\}$ satisfying

(i) $\frac{3}{8} 2^{-n}<r<\frac{7}{8} 2^{-n}$,

(ii) $-\frac{\pi}{8}<\theta<\frac{\pi}{8}$,

(iii) $y$ belongs to the open square centered at $x$ of side length $\frac{7}{8} 2^{-n}$.

Let $\Omega=\Omega[n, x]$ denote the collection of indices $\alpha=\left(n^{\prime}, l^{\prime}, x^{\prime}\right)$ such that $U_{\alpha} \cap V$ is nonempty. Let

$$
W[n, x]=\bigcup_{\alpha \in \Omega} U_{\alpha}, \quad X[n, x]=\bigcup_{n^{\prime} \geqq n} W\left[n^{\prime}, x\right] \quad \text { and } \quad P[n, x]=\left(2^{-(n+1)}, 0, x\right) .
$$

Then,

Theorem 5.12 (Taubes). The gauge transformation s obeys the inequalities

(a) $\sup _{V}\|s-s(P)\| \leqq c\left\|F_{A}\right\|_{L^{2}(W)}$,

(b) $\left|s(P[n, x])-s\left(P\left[n^{\prime}, x^{\prime}\right]\right)\right| \leqq c\left\|F_{A}\right\|_{L^{2}\left(X[n, x] \cup X\left[n^{\prime}, x^{\prime}\right]\right)}$. 
Proof. The inequality (a) follows from a very delicate argument found in Lemma $A_{1}$ of [T]. The inequality (b) follows immediately from (a).

This theorem shows that the sequence $\{s(P[n, x])\}$ converges to some $s_{0} \in S U(2)$ as $n \rightarrow \infty$ and that $s_{0}$ is independent of $x$. Let $s_{0}=J_{\infty}=e^{2 \pi a_{\infty}}$ and $A_{\infty}=a_{\infty} d \theta$ be the constant form.

Lemma 5.13. In any ball $B_{\alpha}$, a point $P$ may be chosen and a radial gauge $\left(A_{r}=0\right)$ found, in which $A(P)=A_{\infty}$ and the inequality,

$$
r^{-2}\left\|A_{\theta}-A_{\infty}\right\|_{L^{q}}+r^{-1}\left\|A_{u}\right\|_{L^{q}}+r^{-1}\left\|A_{v}\right\|_{L^{q}} \leqq \text { const }\|F\|_{L^{q}} \quad \text { for } \quad q \geqq 2,
$$

is satisfied.

Proof. From the results of Sect. 4, we know there is a radial gauge in which $\lim _{r \rightarrow 0} A$ $=A_{\infty}$ a.e. The gauge transformation $g^{\prime}=e^{-A_{u}(P) u}$ gives a new radial gauge $A^{\prime}$ in which $A_{u}^{\prime}(P)=0$. The gauge transformation $g^{\prime \prime}=e^{-A_{v}^{\prime}(P) v}$ now gives a new radial gauge $A^{\prime \prime}$ in which $A_{u}^{\prime \prime}(P)=A_{v}^{\prime \prime}(P)=0$. A constant gauge transformation now fixes $A_{\theta}(P)=a_{\infty}$. In the final normalized gauge,

$$
\left|A_{\theta}-a_{\infty}\right| \leqq \int_{r(P)}^{r}\left|F_{\varrho \theta}\right| d \varrho \leqq \int \varrho|F| d \varrho .
$$

Using Holder's inequality and integrating by parts, we obtain the $L^{q}$ estimate on $A_{\theta}-a_{\infty}$. Estimates on $A_{u}$ and $A_{v}$ are obtained similarly.

Now, let $A$ represent the connection 1-form in the radial gauge of the lemma in the ball containing $U_{0}=U_{(n, 0, x)}$ and let $A_{0}$ be the 1-form in the Coulomb gauge on this same ball. Note the elementary measure theoretic fact that there is a point $Q \in U_{0}$ with $0<\theta_{Q}<\frac{\pi}{2}$ such that

$$
\int_{U_{0} \cap\left(\theta=\theta_{Q}\right)}\left|A-A_{\infty}\right|^{q} r d r d u d v \leqq \frac{2\left\|A-A_{\infty}\right\|_{L^{q}\left(U_{0}\right)}^{q} .}{\frac{\pi}{2}} .
$$

Lemma 5.14. Let $h$ be the gauge transformation on $U_{0}$ from the radial to the Coulomb gauge. Then, $h=e^{-a_{\infty} \theta}\left(I+R_{0}\right)$ where, for $q \geqq 2$,

$$
\left\|R_{0}\right\|_{L^{q}\left(U_{0}\right)} \leqq c r^{2}\|F\|_{L^{q}\left(U_{0}\right)} \text {. }
$$

Proof. Since $d h+A h=h A_{0}$, we may choose $h$ so that

$$
h(\theta)=e^{-a_{\infty} \theta}\left\{I+\int_{\theta_{Q}}^{\theta}\left(h A_{0 \theta}-\left(a_{\theta}-a_{\infty}\right) h\right)+f(r, u, v)\right\},
$$

with $Q$ as defined above, $f(Q)=0$ and

$$
|\nabla f| \leqq \text { const }\left\{\left(\left|A_{u}\right|+\left|A_{v}\right|\right)_{\left(r, \theta_{Q}, u, v\right)}+\int_{\theta_{Q}}^{\theta}\left(\left|A_{0}\right|+\left|A_{u}\right|+\left|A_{v}\right|+r|F|\right)\right\} .
$$

The inequality on the remainder $R_{0}$ follows by taking $L^{q}$ norms, using the measure theoretic fact above, Corollary 5.8, and the Poincare inequality.

If now, $k$ is the gauge transformation on $U_{7}=U(n, 7, x)$ from the radial gauge to the Coulomb gauge, an analogous computation shows that

$$
k=e^{-a_{\infty}(\theta-2 \pi)}\left\{I+R_{7}\right\}
$$

with $\left\|R_{7}\right\|_{L^{q}\left(U_{0}\right)} \leqq c r^{2}\|F\|_{L^{q}\left(U_{0}\right)}$. 
Putting these estimates together,

$$
s=g_{0}^{-1} g_{7}=h^{-1} k=\left(I+R_{0}\right)^{-1} e^{2 \pi a_{\infty}}\left(I+R_{7}\right) .
$$

Lemma 5.15. There exists a point $P$ in $U_{0} \cap U_{7}$ such that

$$
\left|R_{0}(P)\right|<\frac{2 C r^{2}\|F\|_{L^{q}\left(U_{0}\right)}}{\operatorname{meas}\left(U_{0} \cap U_{7}\right)^{1 / q}} \text { and }\left|R_{7}(P)\right| \leqq 1,
$$

where $C$ is the larger of the constants in the bounds on $R_{0}$ and $R_{7}$.

Proof. Let $N=\left\{x \in U_{0} \cap U_{7}|| R_{7}(x) \mid>1\right\}$ and let $m(N)$ be its measure. Then,

$$
m(N)=\int_{N} d x \leqq \int_{N}\left|R_{7}(x)\right|^{q} d x \leqq C r^{2 q}\|F\|_{L^{q}\left(U_{7}\right)}^{q} \leqq C r^{2 q} o(1) .
$$

Since $2 q \geqq 4$, for $r$ sufficiently small,

$$
m(N)<\frac{1}{2} m\left(U_{0} \cap U_{7}\right) \sim r^{4} .
$$

Let $V=\left(U_{0} \cap U_{7}\right) \backslash N$. Then, $\left|R_{7}\right| \leqq 1$ on $V$ and $m(V) \geqq \frac{1}{2} m\left(U_{0} \cap U_{7}\right)$. We can now find a point $P$ in $V$ such that

$$
\left|R_{0}(P)\right|^{q} \leqq \frac{\int_{V}\left|R_{0}\right|^{q} d x}{m(V)} \leqq \frac{2 \int_{V}\left|R_{0}\right|^{q} d x}{m\left(U_{0} \cap U_{7}\right)},
$$

which concludes the proof of Lemma 5.15.

Putting all of these results together, we obtain

Proposition 5.16. The gauge transformation $s=s_{0} e^{z}=g_{0}^{-1} g_{7}$ on $U_{(n, 0, x)} \cap U_{(n, 7, x)}$, where $z \in L_{2}^{q}, 2 \leqq q \leqq p$, and obeys the inequalities,

$$
\begin{gathered}
\|\nabla(\nabla z)\|_{L^{q}}+r^{-1}\|\nabla z\|_{L^{q}} \leqq\|F\|_{L^{q}\left(U_{0} \cup U_{7}\right)}, \\
|z(P)| \leqq \frac{C r^{2}\|F\|_{L^{q}\left(U_{0} \cup U_{7}\right)}}{\left(m\left(U_{0} \cap U_{7}\right)\right)^{1 / q}}
\end{gathered}
$$

where $P=P[n, x]$ is the point of $U_{0} \cap U_{7}$ given by Lemma 5.15.

Proof. The first inequality follows from Corollary 5.8. To obtain the second, note that for small $r,\left(I+R_{0}(P)\right)^{-1}=I-\widetilde{R}_{0}(P)$ with $\left|\widetilde{R}_{0}(P)\right| \leqq 2\left|R_{0}(P)\right|$. Therefore, $s(P)$ $=e^{2 \pi a_{\infty}}-\widetilde{R}_{0}(P)\left(I+R_{7}(P)\right)=s_{0} e^{z(P)} \quad$ with $\left|I-e^{z(P)}\right| \leqq 4\left|R_{0}\right| . \quad$ In other words, $e^{z(P)}=I+W$, with $|W| \leqq 4\left|R_{0}\right|$. Therefore, $|z(P)|=|\log (I+W)| \leqq 2|W| \leqq 8\left|R_{0}\right|$.

Now, let $\lambda(\theta)$ be defined by

$$
\lambda(\theta)=\left\{\begin{array}{lll}
1 & \text { for } & \theta<-\frac{\pi}{16} \\
0 & \text { for } \quad \theta \geqq \frac{\pi}{16}
\end{array}\right.
$$

and note that on $U_{(n, 0, x)} \cap U_{(n, 7, x)},|\nabla \lambda| \leqq c 2^{n}$ and $|\nabla(\nabla \lambda)| \leqq c 2^{2 n}$. We now define the final gauge transformation

$$
\hat{g}=e^{\lambda z} g
$$

The critical term to estimate is

$$
\hat{\omega}=\hat{g}^{-1} d \hat{g}
$$


Using the inequalities of Proposition 5.16, we find that on $U_{0} \cap U_{7}$,

$$
\|\hat{\omega}\|_{L^{q}} \leqq k\|F\|_{L^{q}\left(U_{0} \cup U_{7}\right)} .
$$

It now follows that if $M=\bigcup_{(n, x)}\left(U_{(n, 0, x)} \cap U_{(n, 7, x)}\right.$, then $\|\hat{\omega}\|_{L_{1}^{q}(M)} \leqq 2 k\|F\|_{L^{q}(M)}$.

Using all of the preceding results,

$$
\|\hat{\omega}\|_{L_{1}^{q}\left(N_{0}\right)} \leqq C\|F\|_{L^{q}\left(N_{0}\right)}, \quad 2 \leqq q \leqq p .
$$

Letting $\hat{A}$ be the connection form in the $\hat{g}$ gauge, we see that $\hat{A}=\Gamma+a$, where $\Gamma$ is a flat connection with holonomy $m$ and $a$ satisfies inequality (5.5). This proves Proposition 5.5. Corollary 5.6 follows immediately since $\Gamma=a_{\infty} d \theta$ is a constant flat connection and hence is gauge equivalent to $A^{b}$ by a similarity transformation.

An alternate proof of Theorem 5.1 has been given by Taubes directly in the critical Sobolev case, $p=2$. For completeness, we outline the crucial steps in his argument.

First, he improves the convergence of $s(P[n, x])$ to $s_{0}$ by an iteration. To do this, consider $x$ on the lattice $\wedge_{n}$ of side length $2^{-n-1}$. Projecting $U_{(n, 0, x)}$ onto $\Sigma$ gives a square of side length $2^{-n}$ with center $x$. The square contains four subsquares whose centers lie in $\Lambda_{n+1}$, each of which is the projection onto $\Sigma$ of some $U_{\left(n+1,0, x^{\prime}\right)}$ for $x^{\prime}$ in $\wedge_{n+1}$. Let $Q(x)$ be this set of four points in $\Lambda_{n+1}$. Iterating and using Theorem 5.12, gives

$$
\left|s(P[n, x])-s_{0}\right| \leqq \frac{1}{4} \sum_{x^{\prime}=Q(x)}\left|s(P[n, x])-s\left(P\left[n+1, x^{\prime}\right]\right)\right|+\frac{1}{4} \sum_{x^{\prime}=Q(x)}\left|s\left(P\left[n+1, x^{\prime}\right]\right)-s_{0}\right|
$$

which implies

$$
\left|s(P[n, x])-s_{0}\right| \leqq c\left\|F_{A}\right\|_{L^{2}(W[n, x])}+\frac{1}{4} \sum_{x^{\prime}=Q(x)}\left|s\left(P\left[n+1, x^{\prime}\right]\right)-s_{0}\right| .
$$

Letting $W\left[n, x^{\prime}, k\right]=\{(r, \theta, y)\}$ such that $2^{-n-k-1} \leqq r \leqq 2^{-n-k}$ and $y$ belongs to the projection of $U_{\left(n+k, 0, x^{\prime}\right)}$ for $x^{\prime}$ in $\wedge_{n+k}$; we finally obtain

$$
\left|s(P[n, x])-s_{0}\right|^{2} \leqq c \sum_{k=0}^{\infty} 2^{-k}\left(\left\|F_{A}\right\|_{L^{2}(W[n, x, k])}\right)^{2} .
$$

From these results we now have

Theorem 5.17 (Taubes). The gauge transformation $s=s_{0} e^{z}$ with $z \in L_{2}^{2}\left(N_{0}\right)$ is continuous and

$$
\sup _{V[n, x]}|z| \leqq \sum_{k=0}^{\infty} 2^{-k}\left(\left\|F_{A}\right\|_{L^{2}(W[n, x, k])}\right)^{2} .
$$

The global gauge transformation, $\hat{g}=e^{\lambda z} g$ is continuous and $\hat{\omega}=\hat{g}^{-1} d \hat{g} \in L_{1}^{2}(N)$ and satisfies

$$
\|\hat{\omega}\|_{L_{1}^{2}\left(N_{0}\right)} \leqq c \sum_{(n, x)} \sum_{k=0}^{\infty} 2^{-k}\left(\left\|F_{A}\right\|_{L^{2}(W[n, x, k])}\right)^{2} \leqq c^{\prime}\left\|F_{A}\right\|_{L^{2}\left(N_{0}\right)}
$$

Acknowledgements. We would like to thank Cliff Taubes for suggesting the basic problem and for his many invaluable ideas. We also want to thank Ed Miller for many useful discussions. 


\section{References}

[A] Atiyah, M.F.: Magnetic monopoles in hyperbolic spaces. Vector bundles on algebraic varieties. Tata Institute of Fundamental Research, Bombay (1984), p. 1-33

[B] Braam, P.J.: Magnetic monopoles on three-manifolds. J. Diff. Geom. 30, 425-464 (1989)

[C] Chakrabarti, A.: Spherically and axially symmetric $S U(N)$ instanton chains with monopole limits. Nucl. Phys. B 248, 209-252 (1984)

[F] Floer, A.: Monopoles on asymptotically Euclidean 3-manifolds. Bull. AMS 16, 125-127 (1987)

[FHP $\left.{ }_{1}\right]$ Forgacs, P., Horvath, Z., Palla, L.: An exact fractionally charged self-dual solution. Phys. Rev. Lett. 46, 392 (1981)

$\left[\mathrm{FHP}_{2}\right]$ Forgacs, P., Horvath, Z., Palla, L.: One can have non-integer topological charge. Z. Phys. C 12, 359-360 (1982)

[IZ] Itzykson, C., Zuber, J.B.: Quantum field theory. New York: McGraw-Hill 1980

[JT] Jaffe, A., Taubes, C.: Vortices and monopoles. Progress in Physics, Vol. 2. Boston: Birkhäuser 1980

[O $\left.\mathrm{O}_{1}\right]$ Otway, T.H.: Removable singularities in coupled Yang-Mills-Dirac fields. Commun. Partial Diff. Eq. 12 (9), 1029-1070 (1987)

$\left[\mathrm{O}_{2}\right]$ Otway, T.H.: Higher-order singularities in coupled Yang-Mills-Higgs fields. Nonlinear Anal. 15, 239-244 (1990)

[OS] Otway, T.H., Sibner, L.M.: Point singularities of coupled gauge fields with low energy. Commun. Math. Phys. 111, 275-279 (1987)

[P] Parker, T.: Gauge theories on four-dimensional manifolds. Commun. Math. Phys. 85, 563-602 (1982)

[S] Sibner, L.M.: Removable singularities of Yang-Mills fields in $R^{3}$. Compositio Math. 53, 91-104 (1984)

[SS ${ }_{1}$ Sibner, L.M., Sibner, R.J.: Removable singularities of coupled Yang-Mills fields in $R^{3}$. Commun. Math. Phys. 93, 1-17 (1984)

[SS $\left.\mathrm{SS}_{2}\right]$ Sibner, L.M., Sibner, R.J.: Singular Sobolev connections with holonomy. Bull AMS 19, 471-473 (1988)

[SSU] Sibner, L.M., Sibner, R.J., Uhlenbeck, K.: Solutions to Yang-Mills equations that are not self-dual. Proc. Natl. Acad. Sci. USA 86, 8610-8613 (1989)

[Sm] Smith, P.D.: Removable singularities for Yang-Mills-Higgs equations in two dimensions. Ann. Inst. Henri Poincaré 7, 561-588 (1990)

[T] Taubes, C.H.: Path connected Yang-Mills moduli spaces. J. Diff. Geom. 19, 237-392 (1984)

$\left[\mathrm{U}_{1}\right]$ Uhlenbeck, K.: Removable singularities in Yang-Mills fields. Commun. Math. Phys. 83, 11-29 (1982)

[U $\left.\mathrm{U}_{2}\right]$ Uhlenbeck, K.: Connections with $L^{p}$ bounds on curvature. Commun. Math. Phys. 83, 31-42 (1982)

[W] Warner, F.: Foundations of differentiable manifolds and Lie groups, Scott, Foresman, Glenview, IL (1971). Berlin, Heidelberg, New York: Springer 1984 